\title{
Factorization in hard diffraction
}

\author{
J Collins \\ Physics Department, Penn State University, 104 Davey Laboratory, University Park \\ PA 16802 U.S.A. \\ E-mail: collins@phys.psu.edu
}

\begin{abstract}
In this talk, I reviewed the role of factorization in diffraction hard scattering.
\end{abstract}

\section{Introduction}

I start by reviewing one of the complications that produces seemingly incompatible ways of viewing hard diffraction. This is that some people like the target rest frame, where the dipole picture is most at home, while other people like the Breit frame, where hard-scattering factorization is the natural point of view.

Then I explain what factorization is, and review some of the complications, especially as concerns final-state interactions.

\section{Dipoles v. pdfs in electroproduction}

There are two distinct communities that differ in the reference frame they use and in the associated natural intuitions. The two views have to be compatible, since we are all discussing the same theory and some of the same phenomena. It is important to understand the compatibility of the two views and the strengths and limitations of the intuitive ideas, but this is quite non-trivial. In electroproduction, the views are:

(i) Target rest frame. In this frame the virtual photon is of very high energy, and it normally dissociates into a (virtual) hadronic system long before the interaction with the target. The intuition comes from observing that time dilation implies that the time to cross the proton is much less than the formation time of the hadronic component of the virtual photon:

$$
t_{\text {cross } p} \ll t_{\text {formation of } q \bar{q}} .
$$

Taking the lowest-order, $q \bar{q}$ component gives the dipole model. 
(ii) Breit frame. In this frame, the hard interaction with the virtual photon occurs over a short time and distance scale. The target is fast moving, time-dilated and Lorentzcontracted, so that the hard scattering occurs off a single quasi-free constituent of the target. Thus the transverse distances and times obey

$$
\left(r_{T}, t\right)_{\text {hard sc. }} \ll\left(r_{T}, t\right)_{\text {evolution of target }} \text {. }
$$

A factorization theorem is derived which relates the measured cross section to parton density functions (pdfs).

The predictive power of the dipole model [1] comes from the observation that the transverse structure of the $q \bar{q}$ dipole is frozen as it crosses the target. The important concepts are that of the light-front wave function of the virtual photon and the cross section for the dipole to scatter off the target. The picture is valid at the leading logarithm level, and, by the use of the optical theorem, relations are found between inclusive and diffractive cross sections. The model is also applied to real photon cross sections. Its precise quantitative validity is not clear.

The parton-density point of view gets its power from the observation that just before the hard scattering, the struck quark in the target is separated from the other constituents of the target and may be treated as independent. Important concepts are the number densities of quarks and gluons conditional on the diffracted proton. Also important are the hard-scattering coefficients and the DGLAP evolution of the pdfs, which are the same as in inclusive scattering. The results are valid for the whole leading power in $Q$, not just for the leading logarithms, and the perturbative part is known to at least the first non-leading order.

Of course the cross sections are the same in the two frames. However, the intuition in the two frames is very different. In particular, the time sequence of the scattering is different in the two frames. This is an important symptom that there is non-trivial physics in relating the two points of view. In particular it implies that the struck parton (in the pdf view), before it is struck, must have propagated over a space-like distance, for otherwise the time-sequence would have been preserved under a Lorentz transformation.

This indicates that the process is probing some interesting quantum mechanical properties of QCD related to the EPR phenomenon and Bell's theorem. Although there are definite predictions from the theory, the interpretation of phenomena in terms of a microscopic sequence of cause and effect is frame (and observer) dependent.

The practitioners of the dipole picture gain information about non-perturbative physics by treating dipole cross sections similarly to hadronic cross sections, which gives a lot of useful, if only quantitative information. On the other hand, the practitioners of the pdf point of view are agnostic about non-perturbative physics; but they are able to make various non-trivial predictions from first principles that relate different cross sections. 


\section{Statement of factorization}

Hard-scattering factorization is valid [2] in diffractive deep-inelastic scattering for the diffractive structure functions $F_{2 i}^{(D)}$ themselves, and for subprocesses like jet production and heavy quark production. That is, it is valid for any process for which a factorization theorem is valid in inclusive scattering; diffraction simply differs by a requirement in the target fragmentation region. The factorization theorem for these diffractive processes states that the cross section differential in the relevant variables is of the form

$$
d \sigma=\sum_{i} \int d \xi f_{i}^{(D)}\left(\xi, x_{\mathbb{P}}, t ; \mu\right) d \hat{\sigma}_{i},
$$

up to corrections that are power suppressed in $Q$. Here:

(i) The index $i$ is the flavor of the struck parton.

(ii) The variable $\xi$ is the fractional light-front momentum of the struck parton relative to the "Pomeron", and $t$ and $x_{\mathbb{P}}$ have their usual definitions. Thus $\xi=k^{+} /\left(x_{\mathbb{P}} p^{+}\right)$, where $k$ is the momentum of the struck parton, and $p$ is the momentum of the target.

(iii) The hard-scattering coefficients $d \hat{\sigma}$ are perturbatively calculable and are the same as for the corresponding fully inclusive cross sections.

(iv) The renormalization/factorization scale $\mu$ should be of order $Q$.

(v) The diffractive pdf $f_{i}^{(D)}$ is to be interpreted as the number density of partons conditional on the observation of a diffracted proton in the final state.

(vi) It obeys the standard DGLAP equation for its $\mu$ dependence with the same kernels as for the fully inclusive pdfs.

(vii) The result does not only apply in the diffractive region $x_{\mathbb{P}} \ll 1$, but also at larger values of $x_{\mathbb{P}}$. The diffractive pdfs therefore are the same quantities as the extended fracture functions of Trentadue and Veneziano [3]. Indeed any requirement may be applied in the target fragmentation region, relative to the target, and an obvious generalization of the theorem applies.

The proof of the factorization formula also appears to be valid for the direct photoproduction of jets, heavy quarks, etc. However, as we will see later, the factorization formula fails for hadron-hadron scattering and therefore for resolved photoproduction. (This also implies that the fracture function method fails for the same processes.) Since the separation between direct and resolved photoproduction is scheme-dependent beyond leading order, the quantitative accuracy of factorization in photoproduction is not completely clear.

Originally, the factorization formula was motivated by the model of Ingelman and Schlein [4], who used the common idea that diffraction is due to exchange of a "Pomeron"; they proposed that diffractive hard scattering should be treated as being ordinary hard scattering on a Pomeron target. Associated with this is the concept of parton densities in a Pomeron. Unfortunately, Regge theory in the form used by 
Ingelman and Schlein has not been derived from QCD, and indeed is probably false; this is indicated experimentally by the lack of universality of the Pomeron parameters between different diffractive processes. The factorization theorem that has actually been proved, and is stated above, is somewhat different; it has hard-scattering factorization but not Regge factorization. Non-perturbative Regge properties of the process are in the diffractive parton densities.

The diffractive parton densities have a simple interpretation in terms of light-front creation and annihilation operators of partons. If the ordinary inclusive parton densities are defined as

$$
x f^{\text {usual }}=\int \frac{d^{2} k_{T}}{2(2 \pi)^{3}} \frac{\left\langle p\left|a_{x, k_{T}}^{\dagger} a_{x, k_{T}}\right| p\right\rangle}{\langle p \mid p\rangle},
$$

then the diffractive parton densities are

$$
\frac{x}{x_{\mathbb{P}}} f^{(D)}=\sum_{X} \int \frac{d^{2} k_{T}}{2(2 \pi)^{3}} \frac{\left\langle p\left|a_{x, k_{T}}^{\dagger}\right| X p^{\prime}\right\rangle\left\langle X p^{\prime}\left|a_{x, k_{T}}\right| p\right\rangle}{\langle p \mid p\rangle} .
$$

Here the sum over intermediate states is restricted to those obeying the correct diffractive condition. The extra factor of $1 / x_{\mathbb{P}}$ on the left-hand side of the definition of the diffractive pdf arises because it is convenient to define $f^{(D)}$ as a number density in the fractional momentum of the parton relative to the Pomeron rather than relative to the proton. The factors of $1 /\left[2(2 \pi)^{3}\right]$ are associated with the normalization of the light-front operators.

In these definitions, there are some important complications concerning $U V$ renormalization and particularly concerning the correct gauge-invariant definition of the annihilation and creation operators that I will not go into here.

These formulae can be expressed in terms of quark and gluon fields. For example the gauge-invariant definition of a diffractive quark density is

$f^{(D)}=x_{\mathbb{P}} \sum_{X} \int \frac{d y^{-}}{4 \pi} e^{-i x \mathbb{P}_{\mathbb{P}} \beta p^{+} y^{-}}\left\langle p\left|\bar{\psi}\left(0, y^{-}, 0_{T}\right) P\left(y^{-}\right)^{\dagger}\right| X p^{\prime}\right\rangle\left\langle X p^{\prime}\left|\gamma^{+} P(0) \psi(0)\right| p\right\rangle$.

Here $\psi$ is the ordinary Dirac field for the quark, and $P\left(y^{-}\right)$is the following path-ordered exponential of the gluon field:

$$
P\left(y^{-}\right)=\mathcal{P} \exp \left(-i g \int_{y^{-}}^{\infty} d y^{\prime-} A^{-}\left(0, y^{\prime-}, 0_{T}\right)\right) .
$$

The path-ordered exponential generates an eikonal line in the Feynman-diagram representation of the parton density, and it can be interpreted physically as the struck parton after the hard interaction in the approximation of ignoring its deflection by final-state interactions.

\section{Why is factorization useful?}

(i) Because of the universality of the (diffractive) pdfs between processes, and because of their DGLAP evolution, many predictions can be made from first principles, with the aid of perturbative hard-scattering calculations. 

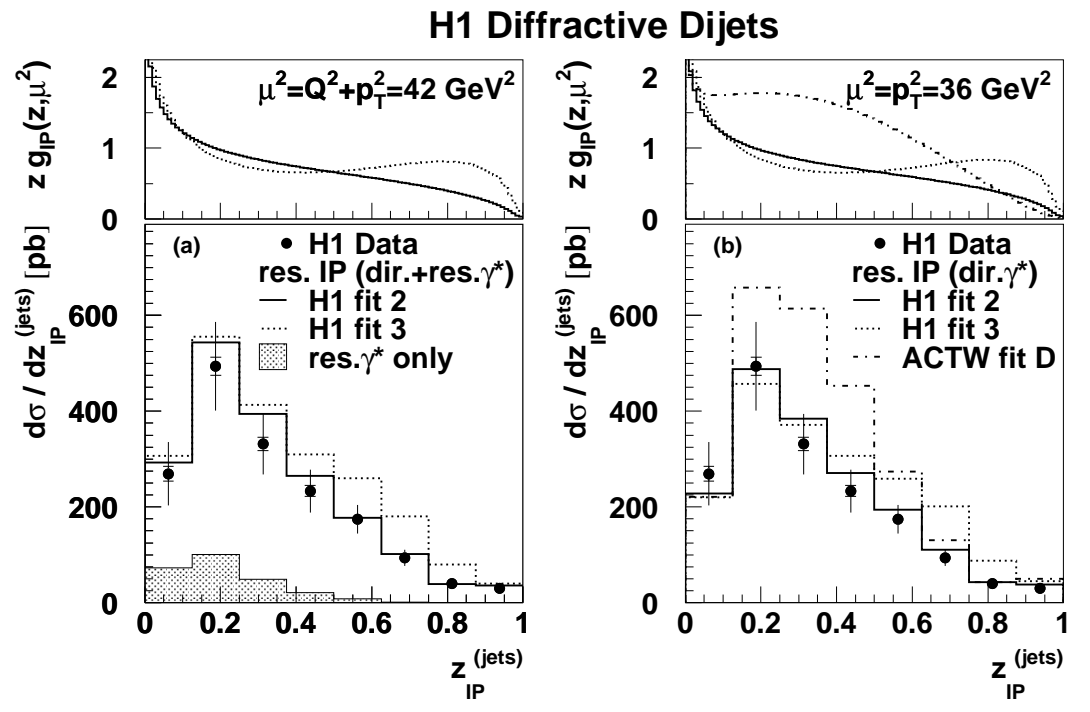

Figure 1. Diffractive electroproduction of jets, from [7]. The variable $z_{\mathbb{P}}^{(\text {jets })}$ is the + component of the dijet's momentum relative to $x_{\mathbb{P}} p^{+}$.

(ii) It isolates the irreducible non-perturbative information in the parton densities alone.

(iii) Hence any treatments of the non-perturbative part of the processes need only concern themselves with the diffractive pdfs alone; they do not need to make separate predictions for $F_{2}^{(D)}$, diffractive heavy quark production, diffractive jet production, etc.

(iv) The theorem is making non-trivial predictions about the dynamics of diffractive processes, in particular about the effect of final-state interactions. The nontriviality of the results is illustrated by the fact that the interactions making the diffracted hadron occur later than the hard scattering and by the lack of factorization in diffractive hadron-hadron scattering.

(v) Since the diffractive gluon density is much larger than the diffractive quark densities [5, 6], diffraction provides a gluon-rich target.

(vi) As mentioned in the previous section, there is an interesting space-time structure for the process. There is an ambiguity as to what causes what. This is likely to have interesting implications for understanding quantum mechanical states in non-perturbative QCD.

Some of the predictions of the factorization theorem are shown in figures 1 and 2 . In these figures, recent data are compared with predictions using fits of diffractive pdfs to other data. The $\mathrm{H} 1$ fits were obtained purely from diffractive $F_{2}^{(D)}$, while the ACTW fits included also the earliest data on direct photoproduction of jets. The predictions work reasonably well, and in particular strongly confirm the need for a large diffractive gluon density. 


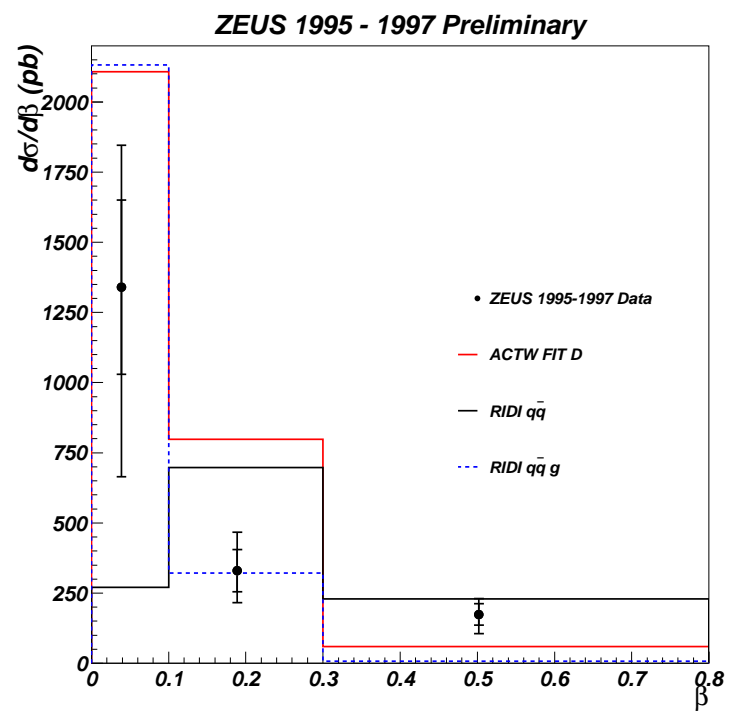

Figure 2. Diffractive electroproduction of charm, from [8].

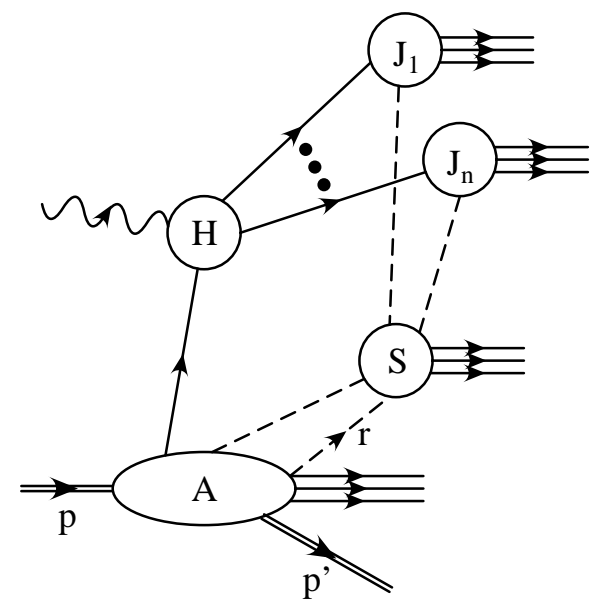

Figure 3. Leading regions for the amplitude for diffractive DIS.

\section{Why is factorization valid [2]?}

The leading regions for the amplitude for diffractive DIS are as shown in figure 3. There the hard subgraph $H$ contains lines all of which are off-shell by order $Q^{2}$ and it results in one or more jets going into the final state. Connected to it by essentially a single parton line is the target subgraph $A$, which consists of lines collinear to the target, and includes the outgoing diffracted proton. Finally, there is a soft subgraph $S$, which consists of lines that are of low energy with respect to both the jet and target subgraphs; it includes the final-state interactions responsible for the neutralization of the color of the partons initiating the jets.

Regions not of this form are power suppressed. Notably, situations in which extra partons are exchanged between the hard subgraph and either the target subgraph or 


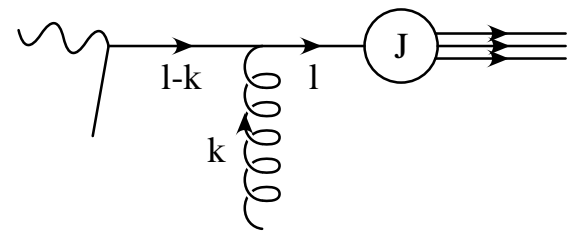

Figure 4. Final-state interaction of jet.

the jet subgraphs are suppressed, because extra lines in the hard subgraph are then far off-shell. Of course, if the gluon density is too high, as in the saturation region, then the large gluon density can overcome this suppression.

The factorization theorem now follows, essentially as in the case of inclusive cross sections - see [2]. However there are a number of complications to note:

(i) Extra exchanges of collinear longitudinally polarized gluons between the hard subgraph and the collinear subgraphs are in fact allowed and unsuppressed, in some gauges, like the Feynman gauge. After an appropriate approximation, we use Ward identities to show that these contributions give the path-ordered exponentials in the gauge-invariant definition of the parton densities.

(ii) This argument does not directly apply to all the final-state interactions, as I now illustrate with the aid of figure 1 . In the most obvious region of quasi-elastic scattering, the exchanged momentum has much smaller longitudinal momentum than transverse momentum: $\left|k^{+} k^{-}\right| \ll k_{T}^{2}$, and the approximations and the Ward identities simply do not apply. However, the only significant dependence on $k^{+}$is in the propagator of the quark line emerging from the hard scattering:

$$
\frac{1}{(l-k)^{2}-m^{2}+i \epsilon} \simeq \frac{1}{-2 l^{-} k^{+}+\text {transverse }+i \epsilon} .
$$

The pole in $k^{+}$is in the upper-half plane, so that the contour of integration can be deformed away from the pole.

The limit to the contour deformation is when $k^{+}$is so big that the dependence of other propagators on $k^{+}$is non-negligible. Then the line $l-k$ is far off-shell, and we have a short-distance interaction, not a genuine final-state interaction, and the previous argument about the longitudinally polarized gluons and the Ward identities applies.

(iii) Even when there are more complicated interactions of the exchanged system and the jet, the singularities are only in the upper-half plane: this corresponds to the fact that the interactions are all in the final-state. Thus the above argument generalizes.

(iv) A slight generalization of the above argument applies when the gluon is soft and obeys $k^{+} k^{-} \sim k_{T}^{2}$. After the use of Ward identities, an application of unitarity is needed before factorization is obtained.

Note that the argument about the final-state interactions is non-perturbative and independent of the diffractive requirement on the cross section. 


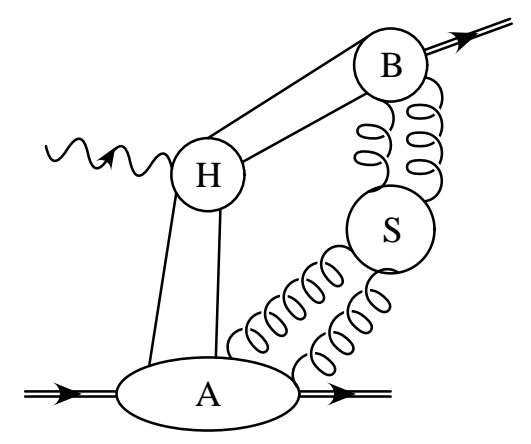

Figure 5. Leading regions for deep-inelastic meson production. The lower pair of quark lines may also be replaced by gluon lines.

\section{Exclusive diffraction}

In this section I consider exclusive diffractive processes, for example deeply virtual Compton scattering (DVCS), which is $e+p \rightarrow e^{\prime}+\gamma+p^{\prime}$ at large $Q$, or inelastic meson production, $e+p \rightarrow e^{\prime}+M+p^{\prime}$, at large $Q$.

The same general idea as before applies [9, 10] to the derivation of a factorization theorem, but now the derivation is applied to the amplitude. For deep-inelastic meson production the leading regions have the form shown in figure 5. The resulting theorem is that the amplitude has the form

$$
\mathcal{M}=\int d \xi \int d z f\left(\xi, x_{\mathbb{P}}, t, Q\right) H(\ldots) \psi(z, Q),
$$

up to power-law corrections. Here $H$ is the perturbatively calculable hard-scattering coefficient, $\psi$ is the light-front wave function of the meson, and $f$ is a skewed parton density. Skewed parton densities [10, 11] are defined just like ordinary parton densities except that they use off-diagonal matrix elements of the quark number operator instead of the expectation values. Schematically, therefore, we have

$$
f=\text { normalization } \int d^{2} k_{T}\left\langle p^{\prime}\left|a_{x, k_{T}}^{\dagger} a_{x, k_{T}}\right| p\right\rangle .
$$

There are some varied conventions concerning the normalization factor. All the same complications concerning final-state interactions, etc, apply as for the factorization theorem for inclusive scattering.

Observe that the cross section is quadratic, not linear, in the skewed parton density.

\section{Lack of factorization in diffractive hadron-hadron scattering}

Factorization does not hold for hard processes in diffractive hadron-hadron scattering, contrary to the case in diffractive deep-inelastic scattering. The problem is that soft interactions between the two hadrons, and their remnants, occur in both the initial and final state. Thus the contour-deformation argument used in diffractive DIS no longer applies. 


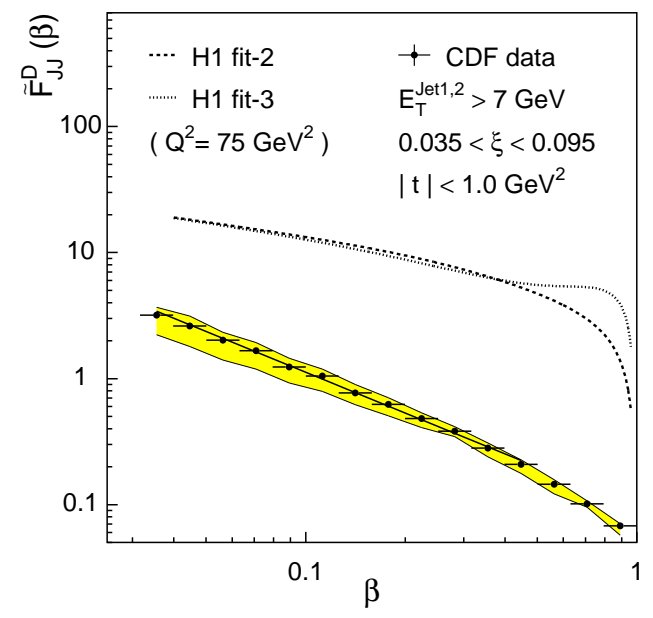

Figure 6. Comparison of CDF's diffractive dijet data with predictions on the basis of factorization and fits to HERA data [6], from [15].

A more difficult argument is needed to give factorization [12], and this only applies to fully inclusive cross sections. Part of the argument involves a unitarity sum over both beam remnants, and this fails in the diffractive case. Even before the discovery of QCD, it was known [13 that factorization fails for diffractive hadron-hadron scattering. In addition, there is another potential mechanism the failure of factorization in QCD, the so-called coherent Pomeron mechanism [14.

The theoretically predicted failure of factorization is in fact observed experimentally. The cross section for diffractive dijet production at CDF [15] is about an order of magnitude below what factorization would predict given the diffractive parton densities measured at HERA - figure 6 .

\section{Conclusions}

- Factorization is proved for diffractive DIS and related processes. It implies a separation of long- and short-distance phenomena, and makes non-trivial predictions.

- The proof involves a non-trivial demonstration that final-state interactions do not affect factorization.

- Factorization fails in diffractive hadron-hadron scattering. This is an experimentally verified consequence of QCD.

- There are non-trivial tests of factorization in diffractive DIS, for example, the prediction of dijet cross sections.

- It is interesting to fully understand the relation between the factorization/pdf approach and the dipole model. 


\section{Acknowledgments}

I would like to thank DESY and the University of Hamburg for their hospitality and the Alexander von Humboldt foundation for an award. This work was supported in part by the U.S. Department of Energy under grant number DE-FG02-90ER-40577.

\section{References}

[1] E.g., McDermott M, Frankfurt M, Guzey V and Strikman V 2000, Eur. Phys. J. C 16, 641 hep$\mathrm{ph} / 9912547$;

Golec-Biernat K and Wüsthoff M 1999, Phys. Rev. D 60, 114023 [hep-ph/9903358].

[2] Collins, J C 1998, Phys. Rev. D 57, 3051 [Erratum-ibid. 61, 019902 (1998)] [hep-ph/9709499].

[3] Grazzini M, Trentadue L and Veneziano G 1998, Nucl. Phys. B 519, 394 hep-ph/9709452].

[4] Ingelman G and Schlein P E 1985, Phys. Lett. B 152, 256.

[5] Alvero L, Collins J C, Terron J and Whitmore J J 1999, Phys. Rev. D 59, 074022 hep-ph/9805268.

[6] Adloff C et al. [H1 Collaboration] 1997, Z. Phys. C 76, 613 [hep-ex/9708016].

[7] Adloff C et al. [H1 Collaboration] 2001, Eur. Phys. J. C 20, 29 [hep-ex/0012051].

[8] Cole J 2000, "Diffractive $D^{* \pm}(2010)$ Production in DIS at HERA", talk at DIS2000 in WG4: http://hep.ph.liv.ac.uk/ disproc/PWG4/.

[9] Collins J C, Frankfurt L and Strikman M 1997, Phys. Rev. D 56, 2982 [hep-ph/9611433].

[10] Radyushkin A V 1997, Phys. Rev. D 56, 5524 hep-ph/9704207.

[11] Müller D, Robaschik D, Geyer B, Dittes F M and Hořejši J 1994, Fortsch. Phys. 42, 101 hep$\mathrm{ph} / 9812448$;

Ji X 1997, Phys. Rev. D 55, 7114 hep-ph/9609381.

[12] Collins J C, Soper D E and Sterman G 1985, Nucl. Phys. B 261, 104;

Bodwin G T 1985, Phys. Rev. D 31, 2616 [Erratum-ibid. D 34, 3932 (1985)];

Collins J C, Soper D E and Sterman G 1988, Nucl. Phys. B 308, 833.

[13] DeTar C E, Ellis S D and Landshoff P V 1975, Nucl. Phys. B 87, 176;

Cardy J L and Winbow G A 1974, Phys. Lett. B 52, 95.

[14] Collins J C, Frankfurt L and Strikman M 1993, Phys. Lett. B 307, 161 hep-ph/9212212.

[15] Affolder T et al. [CDF Collaboration] 2000, Phys. Rev. Lett. 84, 5043. 